\title{
Bonding and Molecular Environment Effects on Near-Infrared Optical Absorption Behavior in Nonlinear Optical Monoazo Chromophore- Polymer Materials
}

\author{
Richard R. Barto ${ }^{a}$ (rick.barto@1mco.com) \\ Curtis W. Frank ${ }^{b}$ (curt.frank@stanford.edu) \\ ${ }^{a}$ Department of Materials Science and Engineering, ${ }^{b}$ Department of Chemical Engineering \\ Stanford University \\ Stanford, CA \\ Peter V. Bedworth (peter.v.bedworth@1mco.com) \\ Rebecca E. Taylor (rebecca.taylor@1mco.com) \\ William W. Anderson (willy.anderson@1mco.com) \\ Susan Ermer (susan.ermer@1mco.com) \\ Lockheed Martin Space Systems Company \\ Advanced Technology Center \\ Palo Alto, CA \\ Alex K-Y Jen (ajen@u.washington.edu) \\ J.D.Luo (jdluo@u.washington.edu) \\ Hong Ma (hma@u.washington.edu) \\ Department of Materials Science and Engineering \\ University of Washington \\ Seattle, WA \\ Hong-Zhi Tang (htang@ncsu.edu) \\ Department of Chemistry \\ North Carolina State University \\ Raleigh, NC \\ Michael Lee (MLee@ApiChip.com) \\ APIC Corporation \\ Culver City, CA \\ Albert S. Ren (no e-mail address available) \\ San Diego, CA
}


Supporting Materials

Table 1. Bake conditions used for PDS film preparation

\begin{tabular}{|l|l|l|l|l|l|}
\hline $\begin{array}{l}\text { Azo dye - polymer } \\
\text { system }\end{array}$ & $\begin{array}{l}\text { Lower bake } \\
\text { temperature } \\
\left({ }^{\mathbf{C}}\right)\end{array}$ & $\begin{array}{l}\text { Lower } \\
\text { bake time } \\
(\mathbf{m i n} .)\end{array}$ & $\begin{array}{l}\text { Ramp } \\
\text { time } \\
(\mathbf{m i n} .)\end{array}$ & $\begin{array}{l}\text { Upper bake } \\
\text { temperature } \\
\left({ }^{\circ} \mathbf{C}\right)\end{array}$ & $\begin{array}{l}\text { Upper } \\
\text { bake time } \\
(\mathbf{m i n} .)\end{array}$ \\
\hline DR1/PMMA guest-host & 130 & 2 & 4 & 215 & 5 \\
\hline DR1/PVP guest-host & 100 & 5 & 5 & 180 & 5 \\
\hline DR19/epoxy guest-host & 130 & 1 & 2 & 215 & $6-7$ \\
\hline $\begin{array}{l}\text { OAcDR1/PMMA guest- } \\
\text { host }\end{array}$ & 100 & 1 & 2 & 160 & $6-7$ \\
\hline $\begin{array}{l}\text { Benzoyl-DR1/PVP guest- } \\
\text { host at 420, 560, and 1060 } \\
\mu \text { mol/g }\end{array}$ & 100 & 5 & 5 & 215 & 5 \\
\hline $\begin{array}{l}\text { Benzoyl-DR1/PVP guest- } \\
\text { host at 1720 } \mu \text { mol/g }\end{array}$ & 100 & 1 & 2 & 215 & 6 \\
\hline $\begin{array}{l}\text { Bis-OAcDR19/epoxy } \\
\text { guest-host }\end{array}$ & 130 & 1 & 2 & 215 & $6-7$ \\
\hline DR1-acrylate copolymers & 100 & 5 & 5 & 200 & 5 \\
\hline DR1-PVP copolymers & 100 & 5 & 5 & 160 & 5 \\
\hline DR19-epoxy copolymers & 100 & 5 & 5 & 160 & 5 \\
\hline
\end{tabular}

Table 2. Spin conditions used for PDS film preparation

\begin{tabular}{|c|c|c|c|}
\hline $\begin{array}{l}\text { Azo dye - polymer } \\
\text { system }\end{array}$ & $\begin{array}{l}\text { Spread } \\
\text { (RPM) }\end{array}$ & Spin Speed (RPM) & Spin Time (seconds) \\
\hline DR1/PVP guest-host & 850 & $1500-1600$ & 40 \\
\hline $\begin{array}{l}\text { Benzoly-DR1/PVP guest- } \\
\text { host at all doping levels } \\
\text { except } 1720 \mu \mathrm{mol} / \mathrm{g}\end{array}$ & 750 & 900 & 20 \\
\hline DR1-acrylate copolymers & $1000-1200$ & $1500-2000$ & 60 \\
\hline DR1-PVP copolymers & $800-930$ & $1330-1380$ & 10 \\
\hline DR19-epoxy copolymers & 800 & 1350 & 60 \\
\hline
\end{tabular}

